\title{
Comparative Assessment of Left Ventricular Function after Coronary Artery Bypass Grafting and Percutaneous Coronary Intervention to LAD by Speckle Tracking Echocardiography Study
}

\author{
Mahmoud S. Abd El Moneum, Eman Saeed Elkeshk, Khaled Emad El-Din El-Rabbat, Adem M. Ahmed \\ Department of Cardiology, Faculty of Medicine, Benha University, Banha, Egypt \\ ORCID: \\ Mahmoud S. Abd El Moneum: https://orcid.org/0000-0002-5639-6521 \\ Eman Saeed Elkeshk: https://orcid.org/0000-0001-9656-3331 \\ Khaled Emad El-Din El-Rabbat: https://orcid.org/0000-0002-1929-7954 \\ Adem M. Ahmed: https://orcid.org/0000-0002-5639-6521
}

\section{Abstract}

Background: Speckle-tracking echocardiography (STE) is a noninvasive imaging technique that analyzes global and regional myocardial function. Aims: This study aims to compare the assessment of the left ventricular (LV) function after percutaneous coronary intervention (PCI). Settings and Design: One hundred patients presented to the catheterization laboratory and cardiothoracic operating room in Benha University Hospital and Kasr Al-Ainy University Hospital for PCI and coronary artery bypass grafting (CABG) to left anterior descending artery (LAD). Patients and Methods: This study enrolled 100 patients. They were divided into two groups: Group I: 50 patients with PCI to LAD and Group II: 50 patients with CABG to LAD. Echocardiography (conventional echocardiography and two-dimensional STE) was performed for all patients $24 \mathrm{~h}$ before and 1 month after the procedures. Statistical Analysis Used: Data management and statistical analysis were done using SPSS version 25. Results: There were significant increases in global circumferential strain (GCS) after CABG surgery with mean value of -17.8 before the procedure and -23.8 after it while there were no significant increases in global longitudinal strain (GLS) after the same procedure with mean value of -17.5 before the procedure and -18.1 after it. There were significant increases in GLS with mean value of -17.0 before PCI and -21.2 after it, and there were significant increases in GCS with mean value of -17.1 before PCI and -25.4 after it with improvement in myocardial function after this procedure. GLS and GCS were significantly higher in patients with PCI than patients with CABG with $P<0.001$. Conclusions: GLS and GCS are more effective than the parameters of standard echocardiography such as the LV end-diastolic volume, LV end-systolic volume, wall motion score index, and ejection fraction for evaluating LV function after PCI and CABG surgery. GLS and GCS are significantly higher in patients with PCI than patients with CABG surgery to LAD.

Keywords: Coronary artery bypass grafting surgery, echocardiography, global circumferential strain, global longitudinal strain, speckle tracing

\section{INTRODUCTION}

Coronary artery disease (CAD) includes a wide spectrum of heart diseases which have the highest mortality in the world ${ }^{[1]}$ Acute myocardial infarction (MI) is a leading cause of morbidity and mortality worldwide. It occurs when irreversible myocardial cell damage or death occurs. ${ }^{[2]}$

\section{Submission: 09-Jul-19 Revision: 18-Jul-19 Accepted: 06-Aug-19}

Published Online: : 25-Nov-19

\begin{tabular}{|l|l|}
\hline \multicolumn{3}{c|}{ Access this article online } \\
\hline Quick Response Code: & Website: \\
& \\
http://www.ijcva.com
\end{tabular}

The management of patients with CADs consists of several approaches, including medical management, percutaneous

\section{Address for correspondence: Dr. Adem M. Ahmed, Department of Cardiology, Faculty of Medicine, Benha University, Benha, Egypt.} E-mail:dradam.ahmed65@gmail.com

This is an open access journal, and articles are distributed under the terms of the Creative Commons Attribution-NonCommercial-ShareAlike 4.0 License, which allows others to remix, tweak, and build upon the work non-commercially, as long as appropriate credit is given and the new creations are licensed under the identical terms.

For reprints contact: reprints@medknow.com

How to cite this article: El Moneum MS, Elkeshk ES, El-Rabbat KE, Ahmed AM. Comparative assessment of left ventricular function after coronary artery bypass grafting and percutaneous coronary intervention to LAD by speckle tracking echocardiography study. Int J Cardiovasc Acad 2019;5:109-15. 
coronary intervention (PCI), and coronary artery bypass grafting $(\mathrm{CABG}){ }^{[3]}$ The use of PCI to manage CAD has expanded significantly during the past three decades. ${ }^{[4]}$

This has resulted in a reduction in mortality rate caused by early and late complications of CAD; however, even successful percutaneous intervention with complete reperfusion to the left anterior descending artery (LAD) does not exclude the risk of left ventricular (LV) remodeling. CABG surgery has been accepted as an effective therapeutic strategy for CAD. ${ }^{[5]}$

CABG does not only improve clinical symptoms but also reduces the risk of sudden cardiac death. It can also increase myocardial function and enhance exercise tolerance. ${ }^{[6]}$ Elderly patients frequently receive $\mathrm{CABG}$; however, they are at high risk because of age-related impairment of organ function and poor surgical tolerance; thus, it is important to accurately evaluate postoperative changes in myocardial function with various parameters. ${ }^{[7]}$

The assessment of the potential benefits of intervention for managing ischemic CAD must include the baseline LV function. ${ }^{[8]}$

The assessment of cardiac function by measuring LV ejection fraction (EF) using echocardiography is the most common method in the daily clinical practice. However, this technique has limitations related with its intra- and inter-observer variability, affected by preload conditions, does not correlate with symptoms and its detection of subtle changes in myocardial function is low. ${ }^{[9]}$

Despite studies evaluating various clinical factors and routine echocardiographic parameters predisposing to LV failure as a complication to CAD, there are still some gaps, and some of the diagnostic parameters failed to identify patients with LV failure after reperfusion. ${ }^{[10]}$

Speckle-tracking echocardiography (STE) is a noninvasive imaging technique that analyzes global and regional myocardial function; STE can overcome some of these limitations. LV global circumferential strain (GCS) (LVGCS) seems to be the most sensitive method to detect myocardial dysfunction and has promise for future applications, ${ }^{[11]} \mathrm{LV}$ global longitudinal strain (GLS) (LVGLS) has been found to be a better parameter to assess LV function than $\mathrm{EF} .{ }^{[12]}$

\section{Aim of the work}

The aim of this study was to compare the assessment of LV function after PCI and CABG to LAD by STE.

\section{Patients and Methods}

The study was conducted on 100 patients who presented to catheterization laboratory and operating room in Benha University Hospital for PCI and CABG to LAD from July 1, 2018, to April 30, 2019 with STE done $24 \mathrm{~h}$ before and 1 month after the procedures.

This study enrolled 100 patients. They were divided into two groups, Group I: 50 patients who had PCI to LAD and Group II: 50 patients who had CABG to LAD.
The patients in this study were divided into 49 patients with ST-elevation MI (STEMI), 36 patients with non-STEMI, and 15 patients with unstable angina without significant difference between the two groups [Table 1].

The patients were at low risk according to The Global Registry of Acute Coronary Events score.

The patients referred to the hospital $48 \mathrm{~h}$ before elective PCI and CABG surgery, the study was done $24 \mathrm{~h}$ before the interventions.

The patients had a history of chest pain before the hospital admission with the following angiographic findings of other epicardial vessels without significant difference between the two groups [Table 2].

Inclusion criteria included patients with LAD lesion proven by previous diagnostic coronary angiography with 50 patients who had PCI to LAD and 50 patients who had CABG to LAD.

Exclusion criteria included patient's refusal, heart muscle disease (severe heart failure and cardiomyopathy). Severe valvular disease, severe myocarditis, significant arrhythmia, previous pacemaker implantation, very poor image quality and uncompleted reperfusion therapy, and patient critical circumflex stenosis.

All patients included in the study were subjected to:

- Informed consent

- Full history taking

- General and local examination of the heart

- 12 lead electrocardiography (ECG)

- Routine laboratory tests including complete blood count, liver functions, renal functions, and virology

- Echocardiography (conventional echocardiography and STE) was performed $24 \mathrm{~h}$ before and 1 month after the procedures.

\section{Conventional echocardiography}

Standard views were used to get images for the LAD territory including:

Parasternal long- and short-axis views at the level of papillary muscle and apical four- and two-chamber views

\section{Table 1: Patient population}

\begin{tabular}{lcc}
\hline & Group I & Group II \\
\hline Patients with STEMI & 20 & 29 \\
Patients with NSTEMI & 22 & 14 \\
Patients with unstable angina & 8 & 7 \\
\hline
\end{tabular}

STEMI: ST-segment elevation myocardial infarction, NSTEMI: Non-STEMI

\begin{tabular}{lcc}
\hline \multicolumn{3}{l}{ Table 2: Population according to coronary angiography } \\
\hline Coronary angiography & Group I & Group II \\
\hline LCX lesion & 25 & 21 \\
RCA lesion & 12 & 15 \\
LCX and RCA & 13 & 14 \\
\hline
\end{tabular}

LCX: Left circumflex artery, RCA: Right coronary artery 
to study the different echocardiographic parameters such as LV end-diastolic volume (LVEDV), LV end-systolic volume (LVESV), wall motion score index (WMSI), and $\mathrm{EF}(\mathrm{EF}=(\mathrm{SV} / \mathrm{EDV}) * 100)$ to compare between the two groups.

\section{Two-dimensional speckle tracking echocardiography}

It is a new technique used for qualification of LV deformation such as GLS and GCS.

GLS is a useful parameter that expresses longitudinal contraction of the myocardium, in adults, GLS $<-16 \%$ is considered abnormal, GLS $>-18 \%$ is considered normal, and GLS $-16 \%--18 \%$ is considered borderline. ${ }^{[13]}$

GCS is used to describe the reduction in the circumference of the LV cavity. Its normal value in adults is ranging from $-20 \%$ to $-27 \%$.

\section{Coronary angiography: Was done to all patients?}

All the echocardiographic studies including offline analysis were done by one competent consultant, and the type of the device used was Vivid 7.

In short-axis views of STE, the circumferential deformation was measured, while the apical views were used to assess longitudinal function. There were no major adverse cardiovascular event during this study.

\section{Statistical analysis}

Data collected throughout history, basic clinical examination, laboratory investigations, and outcome measures were coded; entered; and analyzed using Microsoft Excel software. Data were then imported into the Statistical Package for the Social Sciences (SPSS version 20.0, IBM Company, Armonk, New York, USA) software for analysis. According to the type of data qualitative represent as number and percentage, quantitative continues group represented by mean \pm standard deviation, the following tests were used to test differences for significance. Differences of paired variable were calculated by Mac Namer test, while differences between two groups were calculated by paired t-test. $P<0.05$ were considered significant.

\section{RESULTS}

\section{Study population}

All 100 patients in the two groups consisted of 50 males and 50 females with the mean age $55 \pm 4$ in Group I and $57 \pm 5$ in Group II with 43 patients having diabetes, 63 having hypertension, 57 smokers, 40 having a positive family history, and 41 having dyslipidemia [Table 3 and Figure 1].

\section{Vital signs}

There were no significant differences in pre- and post-procedural systolic blood pressure (SBP) in both groups. $P$ values were 0.491 and 0.828 , respectively.

SBP $\%$ change showed no significant difference between both groups $(P=0.787)$.

\begin{tabular}{lccc}
\hline \multicolumn{3}{l}{ Table 3: General characteristics in both groups } \\
\hline & Group I $(\boldsymbol{n = 5 0 )}$ & Group II $(\boldsymbol{n = 5 0 )}$ & $\boldsymbol{P}$ \\
\hline $\begin{array}{l}\text { Age (years), mean } \pm \text { SD } \\
\text { Gender }\end{array}$ & $55 \pm 4$ & $57 \pm 5$ & 0.141 \\
$\quad$ & & \\
Males & $32(64.0)$ & $28(56.0)$ & 0.414 \\
$\quad$ Females & $18(36.0)$ & $22(44.0)$ & \\
Diabetes & $20(40.0)$ & $23(46.0)$ & 0.545 \\
Hypertension & $31(62.0)$ & $32(64.0)$ & 0.836 \\
Smoking & $33(66.0)$ & $24(48.0)$ & 0.069 \\
Family history & $23(46.0)$ & $17(34.0)$ & 0.221 \\
Dyslipidemia & $17(34.0)$ & $24(48.0)$ & 0.155 \\
\hline Independent $t$-test was used for age. Chi-square test was used for \\
categorical data. SD: Standard deviation
\end{tabular}

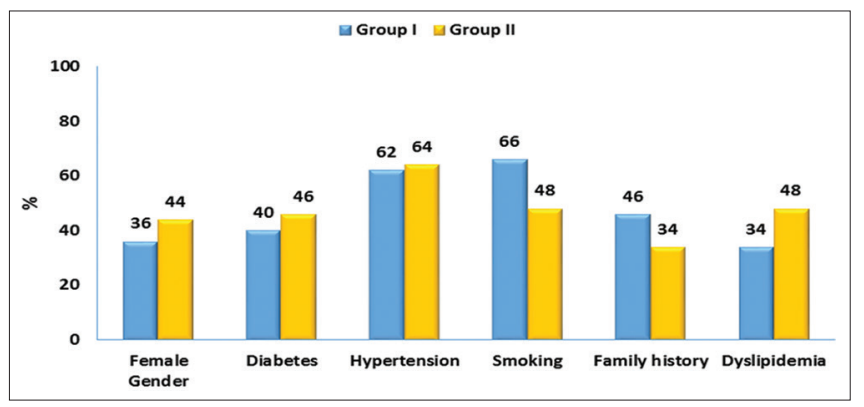

Figure 1: General characteristics in both groups

There were no significant differences in pre- and post-procedural diastolic blood pressure (DBP) in both groups. $P$ values were 0.468 and 0.326 , respectively.

DBP $\%$ change showed no significant difference between both groups $(P=0.207)$.

There were no significant differences in pre- and post-procedural heart rate (HR) in both groups. $P$ values were 0.935 and 0.664 , respectively.

HR \% change showed no significant difference between both groups $(P=0.824)$ [Table 4 and Figure 2].

There were no significant differences between both groups as regards all ECG changes [Table 5 and Figure 3].

\section{Echocardiography}

Conventional echocardiography

The parameters used in conventional echocardiography are LVEDV, LVESV, WMSI, and EF.

There were no significant differences in pre- and post-procedural as regards LVEDV, LVESV, WMSI, and EF in both groups [Table 6 and Figure 4].

Two-dimensional speckle tracking echocardiography

- There were no significant differences in preprocedural GLS between both groups

- Mean postprocedural GLS was significantly higher in Group I compared to Group II

- Median GLS\% change was significantly higher in Group I compared to Group II 


\begin{tabular}{|c|c|c|c|}
\hline & Group I $(n=50)$ & Group II $(n=50)$ & $P$ \\
\hline \multicolumn{4}{|l|}{ SBP } \\
\hline Pre, mean \pm SD & $132 \pm 24$ & $129 \pm 21$ & 0.491 \\
\hline Post, mean \pm SD & $127 \pm 16$ & $128 \pm 11$ & 0.828 \\
\hline Percentage change, median (range) & $0(-22.2-20)$ & $-6.5(-17.6-30)$ & 0.787 \\
\hline \multicolumn{4}{|l|}{ DBP } \\
\hline Pre, mean \pm SD & $86 \pm 12$ & $84 \pm 13$ & 0.468 \\
\hline Post, mean \pm SD & $82 \pm 10$ & $81 \pm 7$ & 0.326 \\
\hline Percentage change, median (range) & $0(-22.2-16.7)$ & $-10.0(-20-41.7)$ & 0.207 \\
\hline \multicolumn{4}{|l|}{ HR } \\
\hline Pre, mean \pm SD & $80 \pm 12$ & $80 \pm 12$ & 0.935 \\
\hline Post, mean \pm SD & $79 \pm 9$ & $80 \pm 8$ & 0.664 \\
\hline Percentage change, median (range) & $0(-17.6-23.1)$ & $0(-22.2-23.1)$ & 0.824 \\
\hline
\end{tabular}

Independent $t$-test was used. Mann-Whitney U-test was used for \% change. SBP: Systolic blood pressure, DBP: Diastolic blood pressure, HR: Heart rate,

SD: Standard deviation

\begin{tabular}{lcc}
\hline \multicolumn{3}{l}{ Table 5: Electrocardiography findings in both groups } \\
\hline ECG & $\begin{array}{c}\text { Group I } \\
(\boldsymbol{n = 5 0 )}, \boldsymbol{n}(\%)\end{array}$ & $\begin{array}{c}\text { Group II } \\
(\boldsymbol{n = 5 0 )}, \boldsymbol{n}(\%)\end{array}$ \\
\hline Normal & $8(16.0)$ & $7(14.0)$ \\
ST depression, T-wave inversion & $22(44.0)$ & $14(28.0)$ \\
ST elevation in anterolateral leads & $20(40.0)$ & $29(58.0)$ \\
\hline \multicolumn{4}{l}{ Chi-square test was used. ECG: Electrocardiography }
\end{tabular}

- There were no significant differences in preprocedural GCS between both groups

- Mean postprocedural GCS was significantly higher in Group I compared to Group II

- Median GCS \% change was significantly higher in Group I compared to Group II [Table 7 and Figure 5].

\section{Coronary angiography}

There were no significant differences between both groups as regards segments of LAD affected $(P=0.204)$ [Table 8].

\section{DISCUSSION}

This study enrolled 100 patients divided into two groups. Group I: 50 patients with PCI to LAD and Group II: 50 patients with CABG to LAD. The aim of this study was to compare the LV function after PCI and CABG to LAD by STE. We revealed that GCS is better than GLS shortly after CABG surgery due to the arrangement of the longitudinal muscle fibers in the LV and the locations of the myocardial regions affected by CAD. Increase in GLS and GCS in patients after PCI with improvement in myocardial function after this procedure. Both GLS and GCS are significantly higher in patients with PCI than patients with CABG surgery to LAD.

In this study, we found that there were no significant differences in terms of age with $P=0.141$, gender with $P=0.414$ and risk factors including smoking, hypertension, diabetes mellitus, dyslipidemia, and family history of coronary heart disease between two groups. This was concordant with D'Andrea et al.

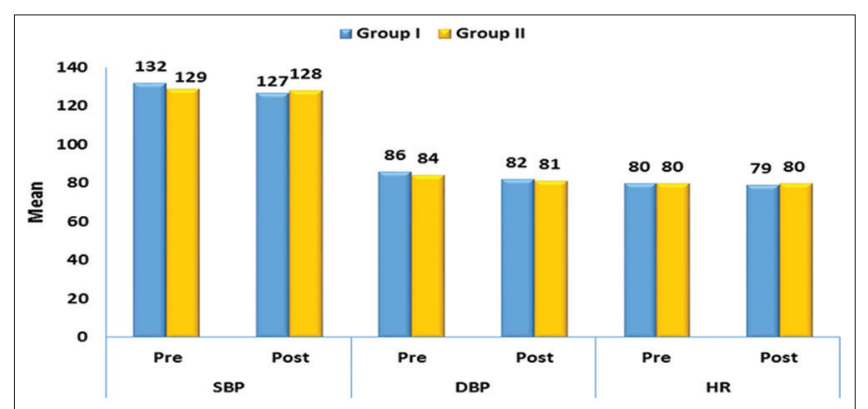

Figure 2: Systolic, diastolic blood pressure, and heart rate in both groups

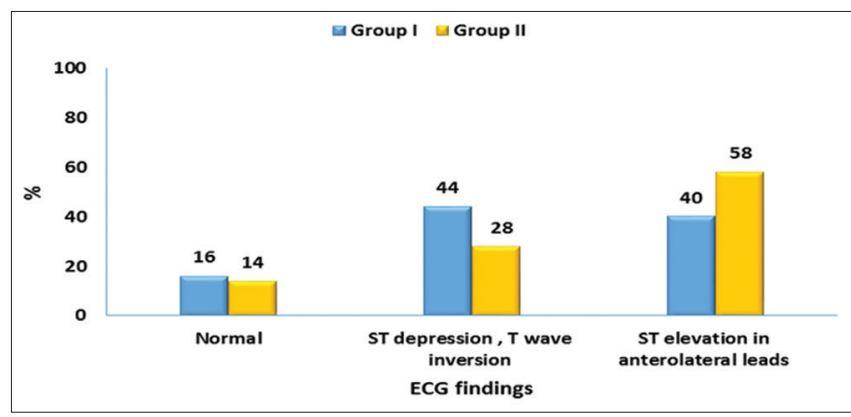

Figure 3: Electrocardiography findings in both groups

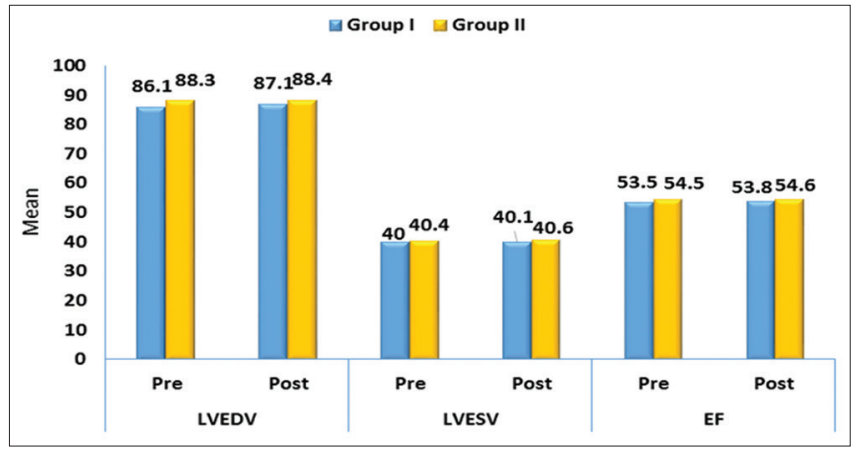

Figure 4: ECHO parameters in both groups

who examined 70 patients with non-ST-segment EMI. ${ }^{[14]}$ This was discordant with Bochenek et al. who examined 66 patients 


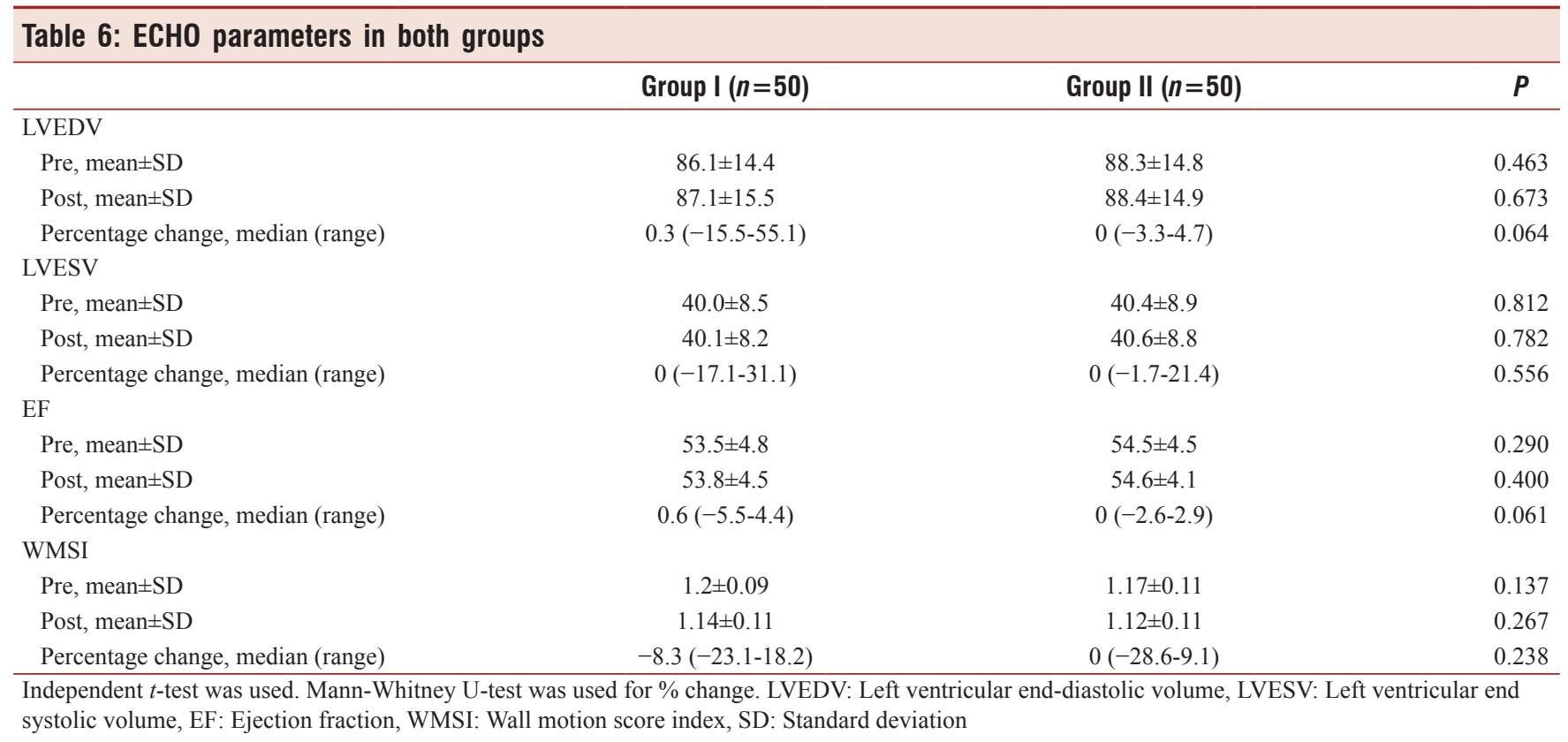

Table 7: Global longitudinal strain and global circumferential strain in both groups

\begin{tabular}{|c|c|c|c|}
\hline & Group I $(n=50)$ & Group II $(n=50)$ & $P$ \\
\hline \multicolumn{4}{|l|}{ GLS } \\
\hline Pre, mean \pm SD & $-17.0 \pm 1.7$ & $-17.5 \pm 1.7$ & 0.142 \\
\hline Post, mean \pm SD & $-21.2 \pm 6.5$ & $-18.1 \pm 1.9$ & 0.001 \\
\hline Percentage change, median (range) & $31.3(9.8-62.9)$ & $3.1(-7.7-12.4)$ & $<0.001$ \\
\hline \multicolumn{4}{|l|}{ GCS } \\
\hline Pre, mean \pm SD & $-17.1 \pm 2.4$ & $-17.8 \pm 2.3$ & 0.124 \\
\hline Post, mean \pm SD & $-25.4 \pm 1.9$ & $-23.8 \pm 2.1$ & $<0.001$ \\
\hline Percentage change, median (range) & $46.7(32.1-100)$ & $33.9(8.9-76.7)$ & $<0.001$ \\
\hline
\end{tabular}

SD: Standard deviation

\begin{tabular}{|c|c|c|c|}
\hline $\begin{array}{l}\text { Coronary } \\
\text { angiography }\end{array}$ & $\begin{array}{c}\text { Group I } \\
(n=50), n(\%)\end{array}$ & $\begin{array}{c}\text { Group II } \\
(n=50), n(\%)\end{array}$ & $P$ \\
\hline Distal & 18 & 15 & 0.204 \\
\hline Mid & 28 & 26 & \\
\hline Proximal & 4 & 9 & \\
\hline
\end{tabular}

Chi-square test was used

with first STEMI $48 \mathrm{~h}$ before and 3 months after primary PCI to evaluate myocardial function. ${ }^{[15]}$

This study demonstrated that there were no significant differences in both SBP and DBP in pre- and post-procedural in the two groups; the $P$ value for SBP was 0.491 and 0.828 in both groups, respectively, while the $P$ value for DBP in both groups was 0.468 and 0.326 , respectively. There were no significant differences in pre- and post-procedural HR in both groups; the $P$ value was 0.935 and 0.664 in the two groups, respectively. In this study, there were no significant differences in ECG in both groups with $P=0.174$.

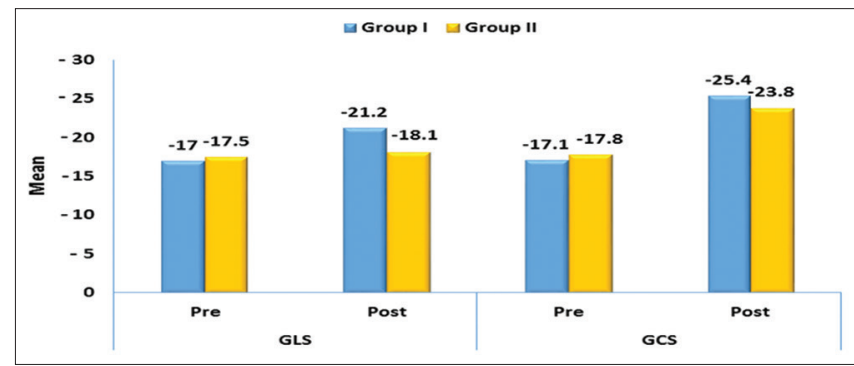

Figure 5: Global longitudinal strain and global circumferential strain in both groups

This was concordant with, Lacalzada et al. who found that there were no significant differences as regarding the vital signs. ${ }^{[16]}$

While GLS mostly reflects subendocardial ischemia, GCS reflects mid-wall ischemia, it is normal that the GLS values are lower than the GCS values and if the subendocardium is affected as in ischemia the GLS will be more affected than the GCS, the normal values of GLS are $-16 \%--18 \%$ while the normal values of GCS are $-20 \%--27 \%$. In our study, after CABG, the GLS takes more time to recover while the 
GCS showed an immediate increase due to the arrangement of muscle fibers.

In this study, we used conventional echocardiography parameters such as LVEDV, LVESV, EF, and WMSI in the both two groups preprocedural and postprocedural.

This study demonstrated that there were no significant differences in LVEDV, LVESV, WMSI, and EF in the both two groups pre- and post-procedural. This finding could have been due to the fact that these parameters are not direct measurements of myocardial function since they are affected by the loading conditions as preload and afterload.

This was in concordance with Gasior et al. who found that there were no significant changes in these parameters; ${ }^{[17]}$ and this was concordant with Shehata who investigated 50 patients with anterior STEMI and found that there were no significant changes in these parameters. ${ }^{[18]}$

This was discordant with Cong et al. who found that echocardiographic parameters such as WMSI were higher in patients with LV remodeling than patients with normal left ventricle after interventions. ${ }^{[19]}$

Two-dimensional STE was used to obtain GLS and GCS in the two groups, preprocedural and postprocedural to compare the LV function in the two groups.

This study demonstrated that there were significant increases in GCS after CABG while there was no significant increase in GLS after the same procedure, this may be related to the arrangement of muscle fibers and the subendocardial ischemia.

This was concordant with Chan et al. who found that there were increases in GCS while there were delays in GLS in patients who undergo $\mathrm{CABG}$ due to the ischemia of longitudinal muscle fibers. ${ }^{[20]}$

This was discordant with Park et al. who investigated 24 patients with STEMI $24 \mathrm{~h}$ before, $48 \mathrm{~h}, 1$ month, 3 months after the procedure and found that there were significant increases in GLS. ${ }^{[21]}$

This study demonstrated that there were significant increases in GLS and GCS in patients after PCI with improvement in myocardial function after this procedure.

This was concordant with Paul and George who found that GLS and GCS were excellent predictors of LV function after primary PCI in patients with acute STEMI. ${ }^{[22]}$

This study demonstrated that both GLS and GCS were significantly higher in patients with PCI than in patients with CABG. This was concordant with Chan et al. ${ }^{[20]}$

After all, STE is recommended to be used in the assessment of LV function after PCI and CABG surgery to LAD to detect the subtle changes after these procedures.

A larger number of studies and longer follow-up period are required to confirm the results and recommendations concerning the assessment of LV function after PCI and CABG surgery in patients with LAD lesion.

\section{Conclusions}

The parameters of STE-GLS and GCS are more effective than the parameters of standard echocardiography such as the LVEDV, LVESV, WMSI, and EF for evaluating myocardial improvements after PCI and CABG surgery. GCS is better than GLS shortly after CABG surgery because of the arrangement of the longitudinal muscle fibers in the LV and the locations of the myocardial regions affected by CAD. Increase in GLS and GCS in patients after PCI with improvement in myocardial function after this procedure. Both GLS and GCS are significantly higher in patients with PCI than patients with CABG surgery to LAD.

\section{Recommendations}

STE is recommended to assess the LV function after PCI and CABG surgery to LAD.

\section{Study limitations}

Some images are suboptimal - small sample size - limited duration of the study.

\section{Financial support and sponsorship}

Nil.

\section{Conflicts of interest}

There are no conflicts of interest.

\section{References}

1. Islam AK, Majumder AA. Coronary artery disease in Bangladesh: A review. Indian Heart J 2013;65:424-35.

2. Kasper DL, Fauci AS, Hauser SL, Jameson JL, Longo DL, Loscalzo J. Harrison's Principles of Internal Medicine. $20^{\text {th }}$ ed: McGraw Hill Education, USA; 2015. p. 98-9.

3. Deb S, Wijeysundera HC, Ko DT, Tsubota H, Hill S, Fremes SE. Coronary artery bypass graft surgery vs. percutaneous interventions in coronary revascularization: A systematic review. JAMA 2013;310:2086-95.

4. Stergiopoulos K, Boden WE, Hartigan P, Möbius-Winkler S, Hambrecht R, Hueb W, et al. Percutaneous coronary intervention outcomes in patients with stable obstructive coronary artery disease and myocardial ischemia: A collaborative meta-analysis of contemporary randomized clinical trials. JAMA Intern Med 2014;174:232-40.

5. Stone GW, Kandzari DE, Mehran R, Colombo A, Schwartz RS, Bailey S, et al. Percutaneous recanalization of chronically occluded coronary arteries: A consensus document: Part I. Circulation 2005;112:2364-72.

6. Saha SK, Kiotsekoglou A, Toole RS, Moggridge JC, Nichols KJ, Govind S, et al. Value of two-dimensional speckle tracking and real time three-dimensional echocardiography for the identification of subclinical left ventricular dysfunction in patients referred for routine echocardiography. Echocardiography 2012;29:588-97.

7. Holmes DR Jr., Kereiakes DJ, Laskey WK, Colombo A, Ellis SG, Henry TD, et al. Thrombosis and drug-eluting stents: An objective appraisal. J Am Coll Cardiol 2007;50:109-18.

8. McGowan JH, Cleland JG. Reliability of reporting left ventricular systolic function by echocardiography: A systematic review of 3 methods. Am Heart J 2003;146:388-97.

9. Marwick TH, Shaw L, Case C, Vasey C, Thomas JD. Clinical and economic impact of exercise electrocardiography and exercise echocardiography in clinical practice. Eur Heart J 2003;24:1153-63.

10. Staron A, Bansal M, Kalakoti P, Nakabo A, Gasior Z, Pysz P, et al. Speckle tracking echocardiography derived 2-dimensional myocardial strain predicts left ventricular function and mass regression in aortic stenosis patients undergoing aortic valve replacement. Int J Cardiovasc Imaging 2013;29:797-808.

11. Lopez-Candales A, Hernandez-Suarez DF. Strain imaging 
echocardiography: What imaging cardiologists should know. Curr Cardiol Rev 2017;13:118-29.

12. Kalam K, Otahal P, Marwick TH. Prognostic implications of global LV dysfunction: A systematic review and meta-analysis of global longitudinal strain and ejection fraction. Heart 2014;100:1673-80.

13. Nagata $Y, W u ~ V C$, Otsuji $Y$, Takeuchi M. Normal range of myocardial layer-specific strain using two-dimensional speckle tracking echocardiography. PLoS One 2017;12:e0180584.

14. D'Andrea A, Cocchia R, Caso P, Riegler L, Scarafile R, Salerno G, et al. Global longitudinal speckle-tracking strain is predictive of left ventricular remodeling after coronary angioplasty in patients with recent non-ST elevation myocardial infarction. Int J Cardiol 2011;153:185-91.

15. Bochenek T, Wita K, Tabor Z, Grabka M, Krzych Ł, Wróbel W, et al. Value of speckle-tracking echocardiography for prediction of left ventricular remodeling in patients with ST-elevation myocardial infarction treated by primary percutaneous intervention. J Am Soc Echocardiogr 2011;24:1342-8.

16. Lacalzada J, de la Rosa A, Izquierdo MM, Jiménez JJ, Iribarren JL, García-González MJ, et al. Left ventricular global longitudinal systolic strain predicts adverse remodeling and subsequent cardiac events in patients with acute myocardial infarction treated with primary percutaneous coronary intervention. Int J Cardiovasc Imaging 2015;31:575-84.

17. Gasior Z, Krejca M, Szmagala P, Bochenek A. Long term left ventricular systolic function assessment following CABG. A prospective, randomised study. Blood versus cristalloid cardioplegia. J Cardiovasc Surg (Torino) 2000;41:695-702

18. Shehata M. Value of two-dimensional strain imaging in prediction of myocardial function recovery after percutaneous revascularization of infarct-related artery. Echocardiography 2015;32:630-7.

19. Cong T, Sun Y, Shang Z, Wang K, Su D, Zhong L, et al. Prognostic value of speckle tracking echocardiography in patients with ST-elevation myocardial infarction treated with late percutaneous intervention. Echocardiography 2015;32:1384-91.

20. Chan J, Hanekom L, Wong C, Leano R, Cho GY, Marwick TH Differentiation of subendocardial and transmural infarction using two-dimensional strain rate imaging to assess short-axis and long-axis myocardial function. J Am Coll Cardiol 2006;48:2026-33.

21. Park SM, Miyazaki C, Prasad A, Bruce CJ, Chandrasekaran K, Rihal C, et al. Feasibility of prediction of myocardial viability with Doppler tissue imaging following percutaneous coronary intervention for ST elevation anterior myocardial infarction. J Am Soc Echocardiogr 2009;22:183-9.

22. Paul A, George PV. Left ventricular global longitudinal strain following revascularization in acute ST elevation myocardial infarction - A comparison of primary angioplasty and streptokinase-based pharmacoinvasive strategy. Indian Heart J 2017;69:695-9. 